\title{
Nanomolecular targeting of dendritic cells for ovarian cancer therapy
}
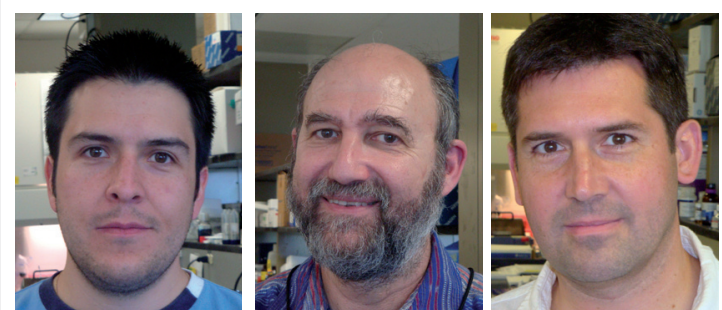

"Improving the very poor

prognosis in most ovarian

cancers will require a combination of novel strategies..."

Juan R Cubillos-Ruiz, Steven Fiering \& Jose R Conejo-Garcia ${ }^{\dagger}$

†Author for correspondence: 640W Borwell, HB 7556, 1 Medical Center Dr, Lebanon, NH 03766, USA = Tel.: +1 6036506223 = Fax: +1 6036506822 = Jose.R.Conejo-Garcia@Dartmouth.edu

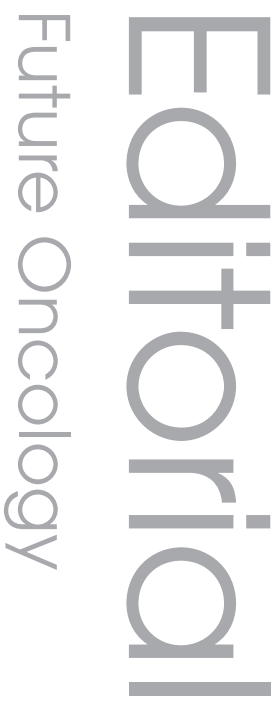

Epithelial ovarian cancer, the most lethal gynecologic cancer in the western world, is responsible for the death of more than 15,000 Americans per year [1]. Despite significant advances in the understanding of tumor cell biology in the last 30 years, current treatments against metastatic carcinoma, the stage at which most ovarian cancers are diagnosed, produce a 5-year survival rate of only $30 \%$. The main challenge for improving the prognosis of ovarian cancer patients is the failure of the current standard approach of surgical debulking followed by chemotherapy to eradicate initially chemosensitive tumors, which leads to development of drug-resistant tumors and systematic relapse [2]. Improving the very poor prognosis in most ovarian cancers will require a combination of novel strategies for early detection and more effective treatments for ovarian cancers diagnosed at a late stage. One area showing promise for improved treatment is developing strategies to foster antitumor immune responses that could block the relapse that generally follows standard treatment.

\footnotetext{
"Despite significant advances in the understanding of tumor cell biology in the last 30 years, current treatments against metastatic carcinoma, the stage at which most ovarian cancers are diagnosed, produce a 5 -year survival rate of only $30 \%$."
}

In recent years, a better understanding of the peculiarities of the ovarian cancer microenvironment has led to novel experimental interventions targeting nontumor cells that are supporting the tumors, an approach that will complement standard chemotherapies [3-5]. These experimental approaches are still at an early stage, but are based on the recognition that epithelial tumors in general and ovarian cancer in particular are composed of multiple cell types. Fibroblasts, endothelial cells and a variety of leukocytes (both antitumor and immunosuppressive) converge at solid tumor locations and within ascites, where they participate in tumor progression.

In this complex ovarian cancer microenvironment, $\mathrm{T}$ cells are the only cell type known to exert spontaneous immune pressure against ovarian cancer progression, to the point that the extent of their infiltration into tumor islets predicts the patient's survival [6-10]. Unfortunately, when ovarian cancers become clinically relevant they have already overcome multiple antitumor immune mechanisms. Several leukocyte subsets recruited by tumors are primarily responsible for suppressing the antitumor immune response. Seminal work by Curiel and colleagues demonstrated a crucial role for regulatory $\mathrm{T}$ cells in tumor-mediated immunosuppression [11]. In addition, our work identified DEC205 ${ }^{+} \mathrm{CD} 11 \mathrm{c}^{+} \mathrm{MHC}-\mathrm{II}^{\text {low }}$ immature dendritic cells (DCs) as the most abundant leukocyte subset in solid tumor specimens in both humans and mouse models [4,12-14]. These DCs act as crucial accomplices in tumor vascularization and immunosuppression $[4,15,16]$. Eliminating immature DCs from tumor locations in experimental models delays ovarian cancer progression by boosting antitumor immunity, which synergizes with conventional chemotherapies and enhances the therapeutic effectiveness of adoptively transferred antitumor $\mathrm{T}$ cells $[3,4,15]$. Ovarian cancer-associated immature DCs therefore emerge as alternative therapeutic targets that may complement current treatments and thus, 
nanomaterials targeting these immunosuppressive leukocytes offer significant promise for future clinical interventions.

The difficulty of selectively targeting nanoparticles to microscopic metastatic tumors via intravenous administration is an obstacle for their clinical implementation. In general, phagocytic leukocytes represent a significant problem for the performance of nanotherapies targeting other cell types since they preferentially ingest nanoparticles. Theoretically, the internalization pathway and the avidity with which tumor-associated leukocytes take up nanomaterials depend on the size, composition and shape of the nanocarrier. Classical phagocytosis describes the uptake of particulate materials that are less than $0.5 \mu \mathrm{m}$, but other endocytic pathways are preferentially used by phagocytic cells to engulf smaller particles [17]. Charged particles of approximately $200 \mathrm{~nm}$ appear to be optimally incorporated by specialized leukocytes [18] although, in most experimental models, even stealth nanomaterials (arbitrarily defined as having one dimension $<100 \mathrm{~nm}$ ) are significantly sequestered by macrophages and DCs [18]. However, active endocytosis by DCs and macrophages can be exploited to load nanoparticles into the abundant immature phagocytic DCs present at the growing edge of solid ovarian cancer masses and in the ascitic fluid.

\section{"Epithelial tumors in general and ovarian cancer in particular are composed of multiple cell types."}

Ovarian cancer is an ideal malignancy for testing novel nanotherapies because, even in late-stage disease, it is frequently restricted to the peritoneal cavity. This allows application of nanoparticles directly into the peritoneum, which avoids the need for systemic nanoparticle delivery required in other forms of cancer, and bypasses the problem of nanoparticle sequestration in the lung and liver. In advanced ovarian cancer, the ascitic fluid irrigates multiple peritoneal tumor masses in which tumor-associated DCs accumulate at the growing edge [4]; therefore, intraperitoneally administered nanomaterials, including siRNApolymer nanocomplexes [14] and superparamagnetic iron oxide nanoparticles [Conejo-Garcia ET aL., Unpublished Data], are preferentially engulfed by $\mathrm{DEC} 205^{+} \mathrm{CD} 11 \mathrm{c}^{+} \mathrm{DCs}$, even in the absence of specific targeting motifs. Ongoing research in our laboratories is currently confirming in experimental models that the preferential uptake of iron oxide nanoparticles by ovarian cancer-associated DCs can be used to induce hyperthermia-mediated killing of these immunosuppressive/proangiogenic cells with magnetic field exposure. This approach could also synergize with the additional targeting of tumor cells with adequately engineered (stealth) nanoparticles, or standard chemotherapies. Drugs encapsulated into liposomes in the nanometer scale or biodegradable polymer-based nanoparticles could also be targeted to both tumor microenvironmental leukocytes and tumor cells.

"Ovarian cancer is an ideal malignancy for testing novel nanotherapies because, even in late-stage disease, it is frequently restricted to the peritoneal cavity."

In addition, because the tumor interstitial matrix is heavily packed with connective tissue and compact accumulations of tumor cells, the penetration of significant amounts of nanomaterials deep into solid tumors, even if they eventually reach the tumor mass, is significantly impaired [19]. A potentially valuable approach to overcome this barrier could take advantage of the spontaneous homing of peritoneal phagocytes to inner solid tumors, where they could act as Trojan horses that would bring the payload to otherwise unreachable areas. For instance, abundant phagocytes in the ascitic fluid engulfing superparamagnetic iron nanoparticles can be used as vehicles to accumulate significant amounts of nanomaterials inside the metastatic masses where they eventually home, and therefore allow hyperthermia-mediated ablation of tumors that otherwise would not contain enough particles.

Rather than using nanoparticles to eliminate tumor-associated immature DCs, nanomaterials can also be engineered to transform the DCs into immunostimulatory DCs that boost the antitumor immune response [20]. Our previous studies demonstrate the capacity of human and mouse DCs from ovarian carcinoma specimens to acquire a mature phenotype in a less suppressive milieu, and to effectively present antigens to specific T cells [12,14-16]. Transforming tumorassociated DCs from an immunosuppressive to an immunostimulatory cell type in vivo and in situ is feasible through the use of nanomaterials and could be applied to generate and sustain spontaneous antitumor responses.

Supporting this concept, our group has recently demonstrated that linear polyethylenimine (PEI)-based nanocomplexes encapsulating 
small interfering RNA (siRNA) are selectively and avidly engulfed by tumor-resident DCs when injected into the peritoneal cavity of ovarian cancer-bearing mice [14]. Extensive analyses determined that engulfment of PEI-based siRNA nanoparticles induced the activation of tumorassociated DCs in vivo in mice and in vitro in human dissociated tumor specimens. This occurred through the combined and nonspecific activation of toll-like receptor (TLR)-7 (by the siRNA oligonucleotides) and TLR5 (by the PEI polymer). DCs incorporating polyplexes became efficient antigen-presenting cells that activated tumor-reactive T cells, which in turn directed tumoricidal activity at the tumors [14]. Mice bearing established ovarian cancers and receiving intraperitoneal injections of PEI-siRNA nanocomplexes underwent T-cell-mediated tumor regression and showed prolonged survival in a completely MyD88-dependent (TLR-dependent) manner [14]. Given the strikingly selective uptake of PEI-based nanocomplexes by leukocytes at ovarian cancer locations (as opposed to tumor cells), and the previously unknown TLR5 agonistic capacity of PEI, it would be interesting to revisit whether or not the significant therapeutic effects observed by many groups in different tumor systems and attributed to silencing of tumor genes via siRNA-PEI, are also a consequence of the selective targeting and activation of tumor-infiltrating phagocytic leukocytes.

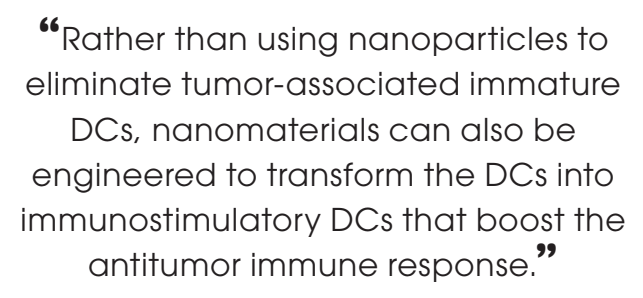

These promising results in preclinical models of aggressive ovarian carcinoma illustrate the potential of exploiting both the phagocytic capacity of tumor microenvironmental cells and the intrinsic immunostimulatory capacity of siRNA-PEI for transforming immunosuppressive cells into Trojan horses that elicit therapeutically relevant immunity at tumor locations. In addition, gene-specific silencing effects of siRNA sequences can be used to downregulate crucial immunosuppressive determinants, which, as we have recently shown, results in discernibly superior therapeutic benefits [14]. The unexpected finding of PEI as a TLR5 agonist suggests that alternative polymers with different chemical compositions that activate different danger sensors may be used to produce nanocomplexes carrying functional siRNA oligonucleotides, which, upon phagocytosis, would target TLRs and activate antigen presention while also suppressing expression of immunosuppressive molecules that these cells express under the influence of tumor cells. Finally, our ongoing studies indicate that ovarian cancer-associated DCs are also capable of engulfing liposomes carrying plasmid DNA encoding T-helper (Th)1-polarizing cytokines, such as interleukin (IL)-18, which are expressed and released to the tumor microenvironment in order to support the activity of antitumor $\mathrm{T}$ cells.

The significant promise of nanotherapies targeting ovarian cancer microenvironmental leukocytes mentioned above is so far only based on results obtained in preclinical models. Nanomaterials may show exciting physical properties, but they will not be applied to solve clinical problems unless they are tested in relevant systems. Many therapies with preclinical promise have not produced useful results when they were applied in patients. The use of preclinical models that reflect the physiopathology of human cancers (including an intact immune system), plus the application of treatments only after the disease is established (as it would be in humans), help make models optimally relevant for the preclinical study of novel nanotherapies. The relative accessibility of peritoneal tumors and prevalence of immunosuppressive and proangiogenic immature DCs makes ovarian cancer an obvious target for the preclinical testing of nanotherapies. In addition, further supporting the applicability of nanoparticle delivery to target ovarian cancer leukocytes, we have demonstrated that different nanomaterials impact the phenotype of the immature DCs contained in dissociated human ovarian carcinoma specimens in vitro in a similar manner as they impact the phenotype of mouse tumor-associated DCs in vivo [14]. Finally, some of the materials utilized in successful experimental models (e.g., PEI and siRNA) are separately being tested in independent clinical trials. This is going to provide very valuable information regarding the safety of these compounds, another issue of paramount importance. Given the paucity of therapeutic options against recurrent ovarian cancers, eliciting therapeutic antitumor immunity through the elimination or modulation of ovarian cancer-associated, immunosuppressive/pro-angiogenic DCs, emerges as an attractive intervention for future trials, either as an individual therapy, or to complement the standard chemotherapeutic approach. 


\section{Financial \& competing interests disclosure}

This publication was supported by a 2006 LizTilberis Award (Ovarian Cancer Research Fund); National Cancer Institute Grants \#R01CA124515 and \#R21CA132026; and Norris Cotton Cancer Center Nanotechnology Group Award. JRCR was supported by the 2009 John $H$ Copenhaver, Jr, and William $H$ Thomas, MD 1952 Fellowship. The authors have no other relevant affiliations or financial involvement with any organization or entity with a financial interest in or financial conflict with the subject matter or materials discussed in the manuscript apart from those disclosed.

No writing assistance was utilized in the production of this manuscript.

\section{Bibliography}

Papers of special note have been highlighted as:

- of interest

-" of considerable interest

1. Jemal A, Siegel R, Ward E et al.: Cancer statistics, 2008. CA Cancer J. Clin. 58(2), 71-96 (2008).

2. Agarwal R, Linch M, Kaye SB: Novel therapeutic agents in ovarian cancer. Eur. J. Surg. Oncol. 32(8), 875-886 (2006).

3. Bak SP, Walters JJ, Takeya M, Conejo-Garcia JR, Berwin BL: Scavenger receptor-a-targeted leukocyte depletion inhibits peritoneal ovarian tumor progression. Cancer Res. 67(10), 4783-4789 (2007).

4. Huarte E, Cubillos-Ruiz JR, Nesbeth YC et al:: Depletion of dendritic cells delays ovarian cancer progression by boosting antitumor immunity. Cancer Res. 68(18), 7684-7691 (2008).

- Demonstrates the detrimental role of tumor-infiltrating dendritic cells (DCs) in ovarian cancer progression and the therapeutic potential of depleting this cell type from the tumor microenvironment.

5. Frederick PJ, Straughn Jr JM, Alvarez RD, Buchsbaum DJ: Preclinical studies and clinical utilization of monoclonal antibodies in epithelial ovarian cancer. Gynecol. Oncol. 113(3), 384-390 (2009).

6. Zhang L, Conejo-Garcia JR, Katsaros D et al:: Intratumoral $\mathrm{T}$ cells, recurrence, and survival in epithelial ovarian cancer. $N$. Engl. J. Med. 348(3), 203-213 (2003).

- Defined for the first time that the extent of intratumoral T-cell infiltration predicts the clinical outcome in ovarian cancer patients.
7. Callahan MJ, Nagymanyoki Z, Bonome T et al.: Increased HLA-DMB expression in the tumor epithelium is associated with increased CTL infiltration and improved prognosis in advanced-stage serous ovarian cancer. Clin. Cancer Res. 14(23), 7667-7673 (2008).

8. Han LY, Fletcher MS, Urbauer DL et al.: HLA class I antigen processing machinery component expression and intratumoral T-cell infiltrate as independent prognostic markers in ovarian carcinoma. Clin. Cancer Res. 14(11), 3372-3379 (2008).

9. Hamanishi J, Mandai M, Iwasaki M et al.: Programmed cell death 1 ligand 1 and tumor-infiltrating CD8 ${ }^{+} \mathrm{T}$ lymphocytes are prognostic factors of human ovarian cancer. Proc. Natl Acad. Sci. USA 104(9), 3360-3365 (2007).

10. Sato E, Olson SH, Ahn J et al:: Intraepithelial $\mathrm{CD}^{+}$tumor-infiltrating lymphocytes and a high $\mathrm{CD}^{+} /$regulatory $\mathrm{T}$ cell ratio are associated with favorable prognosis in ovarian cancer. Proc. Natl Acad. Sci. USA 102(51), 18538-18543 (2005).

11. Curiel TJ, Coukos G, Zou L et al:: Specific recruitment of regulatory $\mathrm{T}$ cells in ovarian carcinoma fosters immune privilege and predicts reduced survival. Nat. Med. 10(9), 942-949 (2004).

12. Conejo-Garcia JR, Benencia F, Courreges MC et al.: Tumor-infiltrating dendritic cell precursors recruited by a $\beta$-defensin contribute to vasculogenesis under the influence of VEGF-a. Nat. Med. 10(9), 950-958 (2004).

- First report demonstrating that immature DCs with pro-angiogenic capacity infiltrate ovarian cancers in order to promote tumor growth.

13. Conejo-Garcia JR, Buckanovich RJ, Benencia F et al.: Vascular leukocytes contribute to tumor vascularization. Blood 105(2), 679-681 (2005).

14. Cubillos-Ruiz JR, Engle X, Scarlett UK et al.: Polyethylenimine-based sirna nanocomplexes reprogram tumor-associated dendritic cells via TLR5 to elicit therapeutic antitumor immunity. J. Clin. Invest. 119, 2231-2244 (2009).

- Shows that intraperitoneally injected siRNA-polyethylenimine nanoparticles are preferentially engulfed by ovarian cancer-associated DCs, a process that transforms them in vivo and in situ from tolerogenic to immunostimulatory cells.

15. Nesbeth Y SY, Cubillos-Ruiz J, Martinez D, Engle X, Turk MJ, Conejo-Garcia JR: CCL5-mediated endogenous anti-tumor immunity elicited by adoptively transferred lymphocytes and dendritic cell depletion. Cancer Res. 69, 6275-6281 (2009).

16. Scarlett U, Cubillos-Ruiz J, Nesbeth Y et al.: In situ stimulation of CD40 and toll-like receptor 3 transforms ovarian cancerinfiltrating dendritic cells from immunosuppressive to immunostimulatory cells. Cancer Res. 69 (18), 7329-7337 (2009).

17. Dobrovolskaia MA, Mcneil SE: Immunological properties of engineered nanomaterials. Nat. Nanotechnol. 2(8), 469-478 (2007).

- Comprehensive review on how nanomaterials can simulate or suppress the host's immune response.

18. Hillaireau H, Couvreur P: Nanocarriers' entry into the cell: relevance to drug delivery. Cell Mol. Life Sci. 66(17), 2873-2896 (2009).

19. Ranney D, Antich P, Dadey E et al:: Dermatan carriers for neovascular transport targeting, deep tumor penetration and improved therapy. J. Control. Release 109(1-3), 222-235 (2005).

20. Demento SL, Eisenbarth SC, Foellmer HG et al: Inflammasome-activating nanoparticles as modular systems for optimizing vaccine efficacy. Vaccine 27(23), 3013-3021 (2009).

\section{Affiliations}

- Juan R Cubillos-Ruiz Department of Microbiology and Immunology, Dartmouth Medical School, Lebanon, NH 03756, USA

- Steven Fiering Department of Microbiology and Immunology, Department of Genetics, Dartmouth Medical School, Lebanon, NH 03756, USA

\section{- Jose R Conejo-Garcia}

Department of Microbiology and Immunology, Department of Medicine, Dartmouth Medical School, Lebanon, NH 03756, USA

Tel.: +1 6036506223

Fax: +16036506822

Jose.R.Conejo-Garcia@Dartmouth.edu 